\title{
Influence of the solar radiation on Earth's climate using the LMDz-REPROBUS model
}

\author{
Sandrine Lefebvre ${ }^{1}$, Marion Marchand ${ }^{1}$, Slimane Bekki ${ }^{1}$, Philippe \\ Keckhut $^{2}$, Franck Lefèvre ${ }^{1}$, Chantal Claud ${ }^{3}$, David Cugnet ${ }^{1}$, \\ Gérard Thuillier $^{2}$ and Alain Hauchecorne ${ }^{2}$ \\ ${ }^{1}$ Université Versailles St-Quentin; UPMC Univ. Paris 06; CNRS/INSU, LATMOS-IPSL, Tour \\ 45-46/4 ${ }^{\grave{e ̀ m}}$ étage, 4 place Jussieu, 75252 PARIS cedex 05, France \\ email: sandrine.lefebvre@latmos.ipsl.fr \\ ${ }^{2}$ Université Versailles St-Quentin; UPMC Univ. Paris 06; CNRS/INSU, LATMOS-IPSL, Route \\ des Gatines, BP 3, 91371 Verrières Le Buisson, France \\ ${ }^{3}$ LMD/IPSL, Ecole Polytechnique, 91128 Palaiseau Cedex, France
}

\begin{abstract}
The atmospheric response to the 11-year solar cycle is studied using the fully interactive 3-D coupled chemistry-general circulation model LMDz-REPROBUS with a complete seasonal cycle. We will show results concerning a comparison between two series of 20-year runs, one in maximum of activity and the other in minimum. The stratosphere-troposphere system shows partly significant response to a solar cycle enhancement of UV radiation. We show how the changes in stratospheric ozone, temperature and zonal wind are connected.
\end{abstract}

Keywords. Methods: numerical, Sun: solar-terrestrial relations

\section{Introduction}

The impact of solar irradiance variations on the terrestrial atmosphere has long been seen as a significant issue. Understanding and quantifying natural climate variability on decadal to centennial time scales is a prerequisite to project future climate changes. Despite the fact that the solar radiation is one of the main drivers for Earth's climate, the mechanisms by which its short-term variation influences atmospheric parameters are controversial and difficult to identify. From a purely energetic point of view, during the 11-year solar cycle, the Total Solar Irradiance (TSI) varies from less than $0.1 \%$. However, much larger fractional changes take place at short wavelengths: for instance, wavelengths less than $400 \mathrm{~nm}$ contribute to about $9 \%$ of the change, and $32 \%$ of the radiation over a solar cycle occur below $250 \mathrm{~nm}$ (Lean 1989). Hence the direct impact of solar irradiance variability is larger in the middle and upper atmosphere than at lower altitudes. Therefore, if there is a solar impact on climate, there must exist processes (maybe depending on the wavelength) that emphasize the effect of the solar cycle.

The interaction of solar radiation with the atmosphere is fundamental in determining its temperature structure and in controlling many of the chemical processes whick take place there. The latter depend on the concentrations and spectral properties of the atmospheric constituents. Photodissociation is an essential component of ozone formation, hence most ozone is produced at low latitudes in the upper atmosphere. Due to transport by the mean meridional circulation, observations show however that ozone is also present in considerable quantities in the mid- and high latitude stratosphere. The stratospheric ozone modulated by the solar cycle could be at the origin of a modulation of the BrewerDobson circulation (Shindell et al. 1999, Haigh 1994 and Haigh 1996). The polar night jet could also be affected as shown by Kodera \& Kuroda (2002), Matthes et al. (2006), 
Matthes et al. (2004) and Haigh \& Blackburn (2006). Solar radiative heating due to the absorption of solar UV through ozone in the upper stratosphere and stratopause regions creates a large temperature gradient during winter, which consequently induces a strong westerly jet (Kodera \& Kuroda 2002).

In this paper, we investigate the atmospheric reponse to the 11-year solar cycle using the fully interactive 3-D coupled chemistry-climate model LMDz-REPROBUS. After briefly describing the model, we will show the first results and a compare with recent analysis of observations.

\section{Stratospheric response to solar forcing}

\subsection{Description of the model LMDz-Reprobus}

LMDz-Reprobus is a coupled chemistry-climate model which includes full representations of dynamical, radiative, and chemical processes in the atmosphere (full chemistry in the stratosphere only) and their interactions, especially feedbacks of the chemical tendencies on the dynamics : in particular, ozone is strongly affected by dynamics and transport. The horizontal grid resolution is $2.5^{\circ} \times 3.75^{\circ}$, there are 50 vertical levels extending from the surface to $0.07 \mathrm{hPa}(\sim 65 \mathrm{~km})$. Details are included in Lefèvre et al. (1994), Lefèvre et al. (1998), Lott et al. (2005) and Jourdain et al. (2008). Solar variability is forced explicity in the models through changes in the photolysis rates.

\subsection{Experimental setup}

The goal here is to evaluate the model response to the 11-year solar cycle. We have done simple time-slice experiments with solar fluxes from the SUSIM $\dagger$ data: two series of 20-year runs, one in maximum of activity (chosen as February 2, 1992) and the other in minimum (chosen as October 10, 1996) are computed. Figure 1 shows the irradiance signal for both chosen dates and their ratio: note the non negligeable value of the ratio (up to $20 \%$ ) in the UV band 140-240 nm.
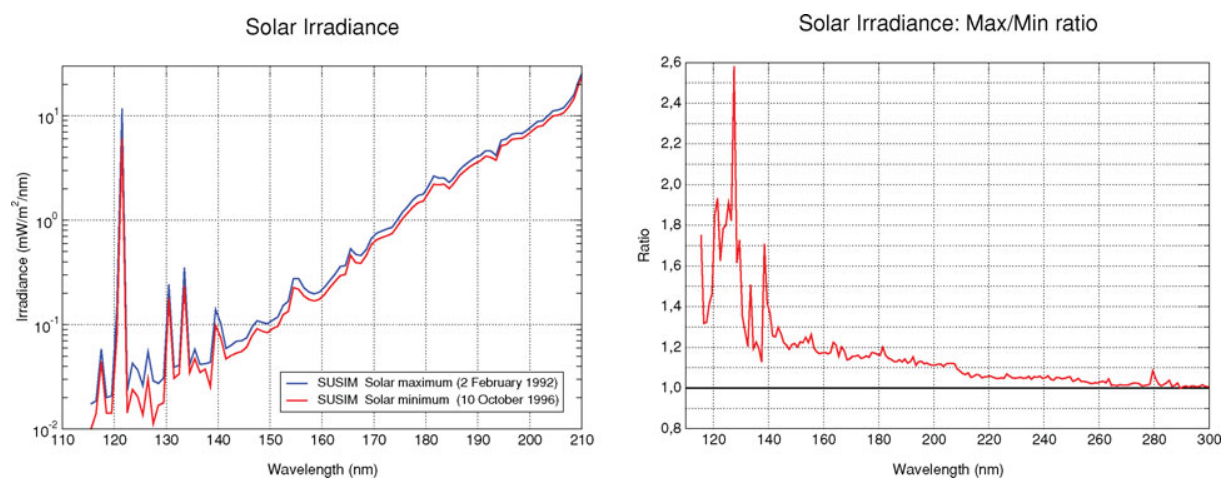

Figure 1. Left: Variation of the SUSIM solar irradiance for the chosen dates February 2, 1992 and October 10, 1996. Right: Ratio of both signal max/min.

\subsection{Results}

Preliminary results for the temperature, the zonal wind and the ozone concentration are shown in Figures 2 and 3. These graphs present a comparison between both series by

$\dagger$ Solar Ultraviolet Spectral Irradiance Monitor 

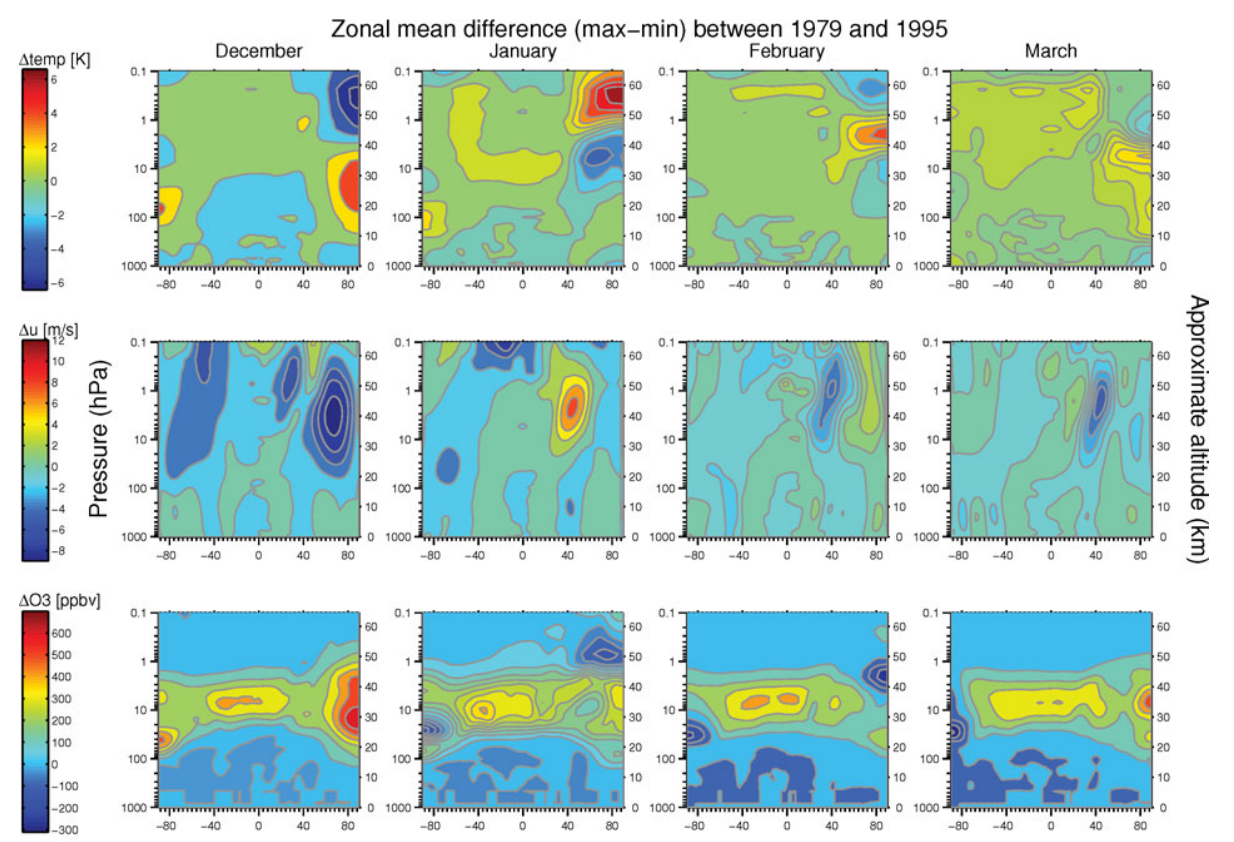

Latitude (degrees)

Figure 2. Zonal mean difference (max-min) for the temperature temp (top), the zonal wind $u$ (middle) and the ozone concentration $\mathrm{O}_{3}$ (bottom), in the atmosphere for the northern hemisphere winter months (December to March).

computing the mean difference max-min. We plotted panels respectively for the northern hemisphere winter months where the solar signal is strongly zonally asymmetric, and for the southern hemisphere winter months. The stratosphere is generally warmer of about 0.5 to $1 \mathrm{~K}$ at maximum, accompanied by a slight cooling at low latitude in the troposphere, suggesting a dynamical origin. At higher latitudes, the response is well marked with a reversal of the temperature difference near the stratopause level in both hemisphere. The temperature anomalies are associated with zonal wind anomalies for the same months: in austral winter, the zonal wind anomaly peaking at $40^{\circ}$ and at $2 \mathrm{hPa}$ is always positive, meaning a reinforcement of the austral polar vortex. This is not the case in boreal winter, where there is a positive anomaly of the zonal wind in January, so a stronger polar vortex during this month, whereas this anomaly becomes strongly negative during the other months, meaning a destabilization of the boreal polar vortex. For clarity of the graphs, we did not plot the significance levels but all these strong anomalies are statistically significant at 95\%. A clear ozone increase is visible in the stratosphere at solar maximum, with a peak at $10 \mathrm{hPa}$ in the equatorial band. There are two negative anomalies at high latitude at about $20 \mathrm{hPa}$ and $1 \mathrm{hPa}$ in January and February in the winter season, but only one peaking at $2-3 \mathrm{hPa}$ in summer, and a positive one centered around $10 \mathrm{hPa}$ in December and March.

\section{Comparison with observations}

These results correspond to a reinforcement of the polar winter vortex above the Antarctic but the behavior is different above the Arctic with a stronger polar vortex in January and a destabilization in February, under solar maximum conditions (SMC). Now if we do a comparison with recent observations, first the temperature response is 

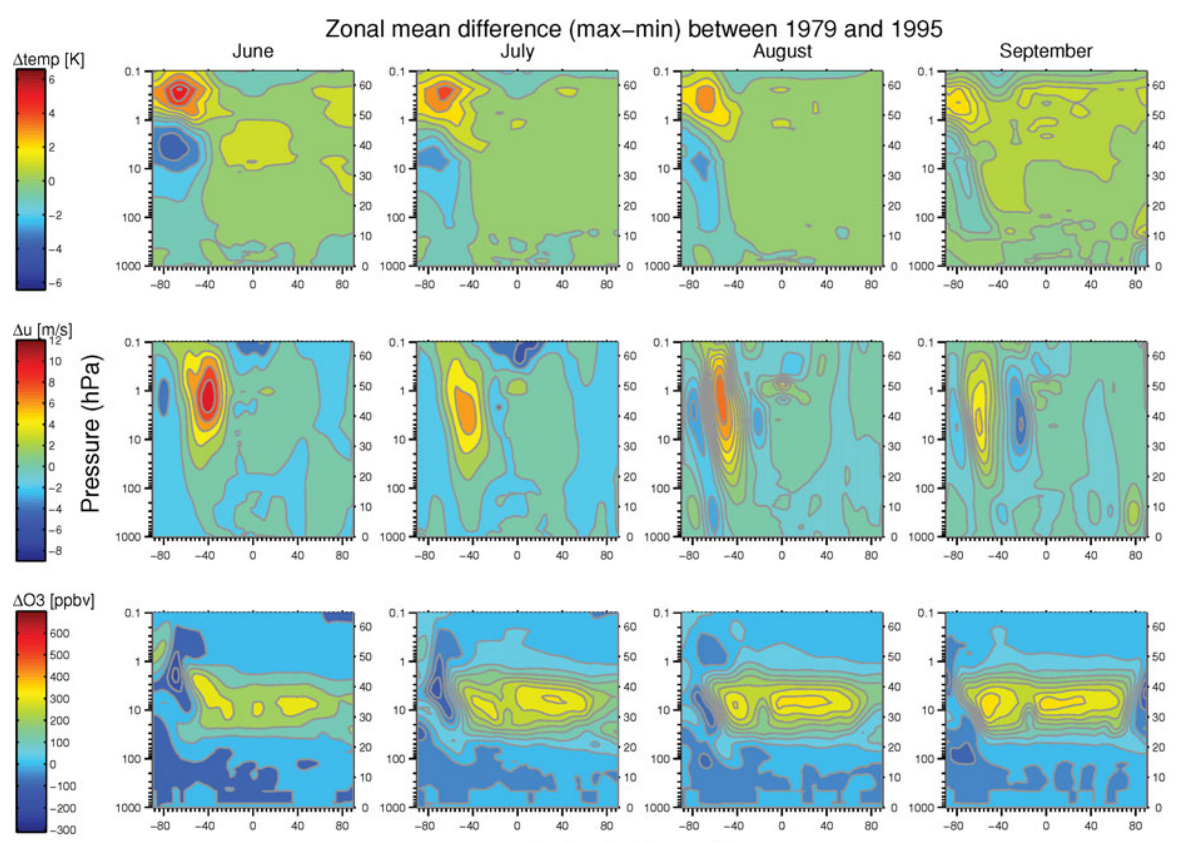

Latitude (degrees)

Figure 3. Same as Figure 2 but for the southern hemisphere winter months (June to September).

consistent with the observations in the upper stratosphere-lower mesosphere (direct effect on ozone) as shown in Figure 4 from Keckhut et al. (2005). Indeed, curves in left panel confirm a significant sign change near $50 \mathrm{~km}$ during winter at high northern latitude. Moreover the right panel clearly shows a strong seasonal behavior at mid-and highlatitude with a maximum response around $50^{\circ}$ in both hemispheres while at low latitudes the response is more slowly changing. The high latitude response ranges from a slightly positive reponse to a large negative one. The later extends from October to April in the northern hemisphere with a maximum amplitude of $-1.2 \mathrm{~K}$, while it extends from May to september in the Antarctic with an amplitude of $-2 \mathrm{~K}$. Our runs exhibit also a strong negative response in the southern hemisphere during austral winter. These results confirm that both hemisphere have a different response in term of amplitude, extension, and timing.

Secondly, by a study using satellite SSU† temperature measurements and ERA-40 zonal winds, Claud et al. (2008) also demonstrated that temperatures are generally warmer for low- and mid-latitudes under SMC, and that, at northern high latitude, the polar vortex is stronger with the exception of February and to a lesser extent March in the northern hemisphere. Indeed, Figure 5 shows that, for latitudes north of $50^{\circ} \mathrm{N}$, there is a significant negative signature in early winter, then in February-March the sign reverses, before returning to negative from April onward. While in December, SMC are associated with colder temperatures practically all along the vertical, in January, a warming in the low stratosphere appears. In February, north of $50^{\circ} \mathrm{N}$, temperatures are warmer over the whole vertical range (significantly from 50 to $5 \mathrm{hPa}$ ), and temperatures are colder in the equatorial region below $20 \mathrm{hPa}$. In March, SMC are again associated with

$\ddagger 40$ year Re-Analysis Data Archive of the European Centre for Medium-Range Weather Forecasts 

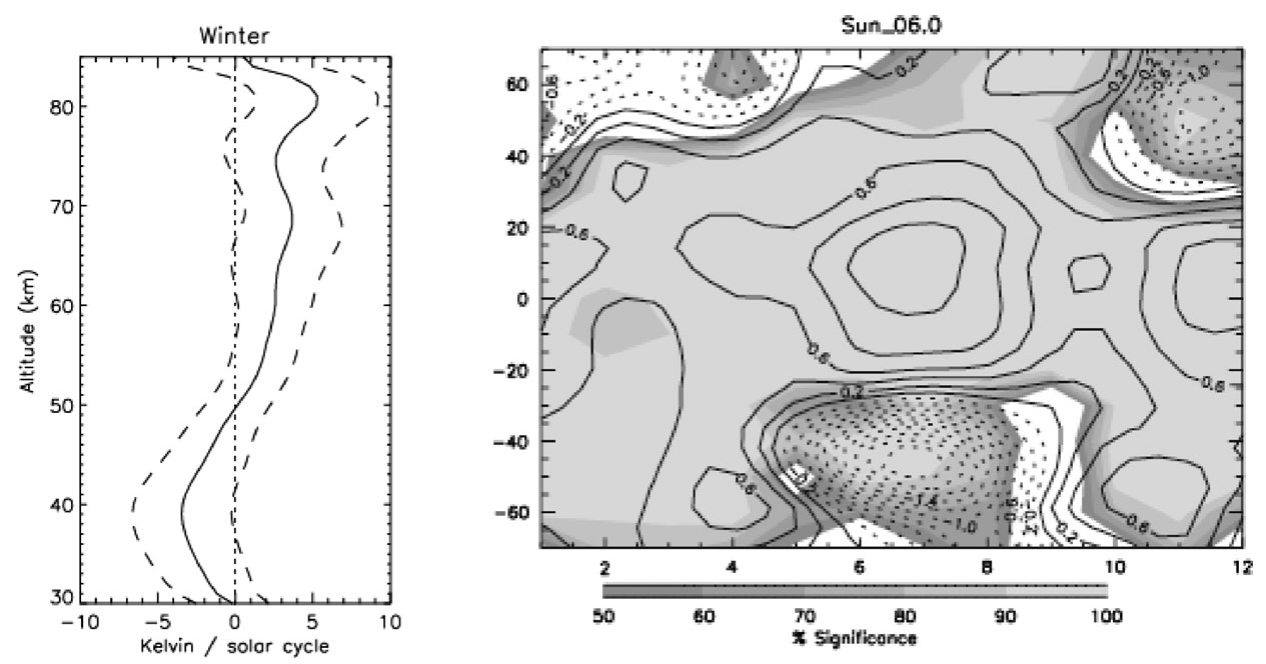

Figure 4. Left: Temperature solar response as a function of altitude deduced from lidar data obtained since 1979 up to 2001 at Observatory of Haute Provence $\left(44^{\circ} \mathrm{N}\right)$, averaged over the 6 northern winter months (October-March). Right: Monthly zonal temperature response to solar activity based on SSU/MSU data from 1979 up to 1998 and represented as a function of latitudes and months for a given height level corresponding to $6 \mathrm{hPa}$. The gray shaded regions indicate statistically significant signal (from Keckhut et al. 2005).
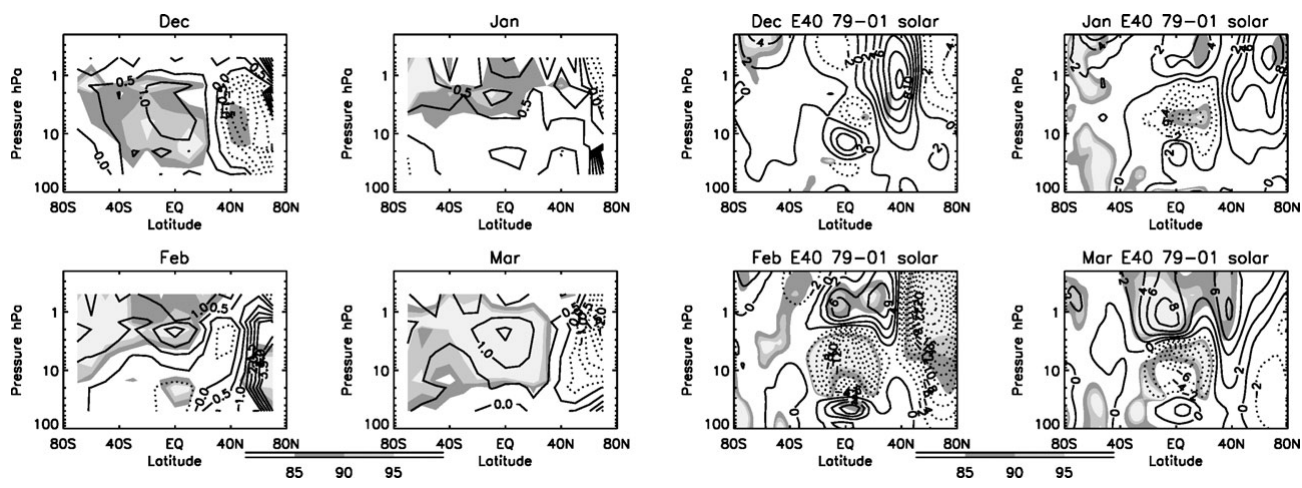

Figure 5. Left: SSU temperature measurements from radiometer on board NOAA satellites between 1979 and 2004: zonal temperature response (in K/solar cycle) to solar activity for the winter-early spring of the northern hemisphere. The shades of gray correspond to significance level. Contours are every $0.5 \mathrm{~K} /$ solar cycle. Right: ERA-40 wind measurements betweeen 1979 and 2001: zonal wind response (in $\mathrm{m} / \mathrm{s} /$ solar cycle) to solar activity for the winter-early spring of the northern hemisphere. The shades of gray correspond to significance level. Contours are every $2 \mathrm{~m} / \mathrm{s} /$ solar cycle (from Claud et al. 2008).

a cooling of the polar vortex. Figure 5 shows a positive zonal wind anomaly in the northern hemisphere that appears in december above $10 \mathrm{hPa}$, and developing in January, consistent with a stronger polar vortex in solar maximum. In February, a negative significant anomaly is observed north of $40^{\circ} \mathrm{N}$ indicating that this month the solar maximum is associated with a weaker vortex. The analysis on the zonal wind then reports a stronger polar vortex during solar maximum, associated with a latitudinal temperature gradient at the stratopause that is evident in December and in January, but not in February and partially in March. 


\section{Discussion and conclusions}

The present study shows how the stratosphere-troposphere system partly responds significantly to a solar cycle enhancement of UV radiation. But the associated mechanism is currently unknown even if ideas has been suggested in the literature.

The main idea is that the mechanism is maybe linked to the interaction of the planetary waves with the main circulation. In particular, Kodera \& Kuroda (2002) has argued that changes in the winter polar stratosphere brought about by anomalous solar heating may influence the passage of upward propagating planetary waves and thus their deposition of momentum that influences the strength of the mean overtuning of the stratosphere. Gray et al. (2001) also demonstrated that zonal wind anomalies in the sub-tropical upper stratosphere can influence the timing and amplitude of sudden stratospheric warmings, events during the polar winter in which enhanced planetary wave activity disturbs the cold polar vortex.

Perspectives of this work consist in the computation of the Eliassen-Palm fluxes which will permit to investigate the interaction with the planetary waves. Moreover, in a next future is planned the analysis of a run with a real solar signal as input for the irradiance.

This work is led in the framework of the PICARD mission, whose launch is scheduled at the beginning of 2010 . This satellite is devoted to a better knowledge of (i) the physics and internal structure of the Sun, and (ii) the solar forcing on Earth's climate (see http://smsc.cnes.fr/PICARD/index.thm and Thuillier et al. 2006)

\section{Acknowledgements}

S. Lefebvre is supported by a CNES post-doctoral grant.

\section{References}

Claud, C., Cagnazzo, C., \& Keckhut, P. 2008, JASTP, 70, 2031

Gray, L. J., Phipps, S. J., Dunkerton, T. J., et al. 2001, Quart. J. R. Meteo. Soc., 127, 1985

Haigh, J. D. 1994, Nature, 370, 544

Haigh, J. D. 1996, Science, 272, 981

Haigh, J. D. \& Blackburn, M. 2006, Space Sci. Revs, 125, 331

Jourdain, L., Bekki, S., Lott, F., \& Lefèvre, F. 2008, Ann. Geophys., 26, 1391

Keckhut, P., Cagnazzo, C., Chanin, M.-L., Claud C., \& Hauchecorne A. 2005, JASTP, 67, 940

Kodera, K. \& Kuroda, Y. 2002, JGR, 107(D24), 4749

Lean, J. L. 1989, Science, 244, 197

Lefèvre, F., Brasseur, G. P., Folkins, I., Smith, A. K., \& Simon, P. 1994, JGR, 99, 8183

Lefèvre, F., Figarol, F., Carslaw, K. S., \& Peter, T. 1998, GRL, 25(13), 2425

Lott, F., Fairhead, L., Hourdin, F., \& Levan, P. 2005, Clim. Dyn., 25, 851

Matthes, K., Langematz, U., Gray, L. J, Kodera, K., \& Labitzke, K. 2004, JGR, 109, D06101

Matthes, K., Kuroda, Y., Kodera, K., \& Langematz, U. 2006, JGR, 111, D06108

Shindell, D., Rind, D., Balachandran, N., Lean, J., \& Lonergan, P. 1999, Science, 284, 305

Thuillier, G., Dewitte, S, \& Schmutz, W. 2006, Adv. Sp. Res, 38, 1792 